\title{
Medical Microbiology and Immunology for Dentistry
}

Author: Nejat Düzgünes

Publisher: Quintessence Publishing

Language: English

ISBN: 978-0-86715-647-8

Edition: $1 / \mathrm{e}$

Publish Year: 2016

Pages: 306, illustrated

Price: $118.00 €$

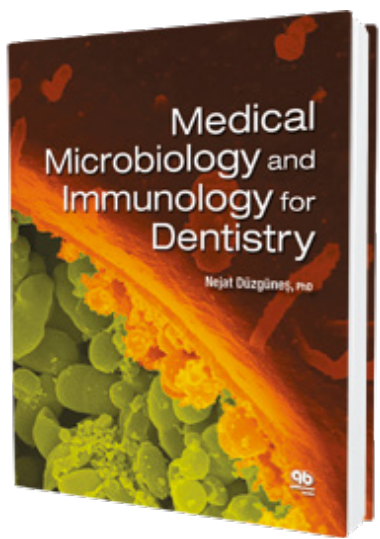

Nejat Düzgünes, PhD, currently Professor in the Department of Biomedical Sciences at the University of the Pacific Arthur A. Dugoni School of Dentistry decided to write this book entitled Medical Microbiology and Immunology for Dentistry specifically addressed to dentists, because all medical microbiology textbooks are generally much too detailed for training purposes. This textbook has five parts and 41 chapters. The first part is dedicated to Immunology and presents the immune system, antibodies and complement, immune response and vaccines. The second part is about Bacteria, and talks about bacterial structure, metabolism, and genetics, bacterial pathogenesis, antibacterial chemotherapy, sterilization, disinfection, and antisepsis, microbial identification and molecular diagnostics and illustrates a lot of bacterial types. Oral microbiology is covered in two major chapters: Oral Microflora and Caries, and Periodontal and Endodontic Infections. A discussion about Fungi, fungal structure, replication, and pathogenesis, fungal diseases and antifungal chemotherapy is the content of part three. The last two parts present Viruses, Prions and Parasites along fourteen chapters. Most of the conditions treated by dentists are the result of bacterial infection and understanding the microbiology of these diseases is essential for their treatment. Through complete information on microbiology and immunology, this book is a quintessence of specialty literature and a therapeutic guide useful in each dentist's daily practice.

DOI: 10.25241/stomaeduj.2017.4(4).bookreview.2 